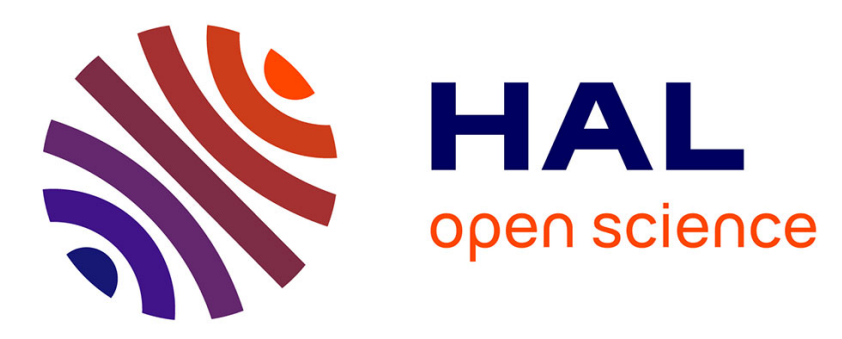

\title{
Reliability Improvement of Odour Detection Thresholds Bibliographic Data
}

\author{
Pascale Montreer, Stefan Janaqi, Stéphane Cariou, Mathilde Chaignaud, \\ Isabelle Betremieux, Philippe Ricoux, Frédéric Picard, Sabine Sirol, Budagwa \\ Assumani, Jean-Louis Fanlo, et al.
}

\section{To cite this version:}

Pascale Montreer, Stefan Janaqi, Stéphane Cariou, Mathilde Chaignaud, Isabelle Betremieux, et al.. Reliability Improvement of Odour Detection Thresholds Bibliographic Data. IPMU 2018 17th International Conference on Information Processing and Management of Uncertainty in Knowledge-Based Systems., Jun 2018, Cadiz, Spain. pp.562-573, 10.1007/978-3-319-91473-2_48 . hal-01955230v1

\section{HAL Id: hal-01955230 \\ https://hal.science/hal-01955230v1}

Submitted on 14 Dec 2018 (v1), last revised 17 Jun 2022 (v2)

HAL is a multi-disciplinary open access archive for the deposit and dissemination of scientific research documents, whether they are published or not. The documents may come from teaching and research institutions in France or abroad, or from public or private research centers.
L'archive ouverte pluridisciplinaire HAL, est destinée au dépôt et à la diffusion de documents scientifiques de niveau recherche, publiés ou non, émanant des établissements d'enseignement et de recherche français ou étrangers, des laboratoires publics ou privés. 


\title{
Reliability Improvement of Odour Detection Thresholds Bibliographic Data
}

\author{
MONTREER P. ${ }^{1^{*}}$, JANAQI S. $^{2}$, CARIOU S. ${ }^{1^{\circ}}$, CHAIGNAUD M. ${ }^{3 *}$, BETREMIEUX \\ $\mathrm{I}^{4^{*}}$, RICOUX P. ${ }^{4^{\circ}}$, PICARD F. ${ }^{5}$, SIROL S..$^{6^{*}}$ ASSUMANI B. ${ }^{\circ}$, and FANLO J.L..$^{1,3^{\circ}}$. \\ ${ }^{1}$ IMT Mines Alès, LGEI laboratory, 6 av. de Clavières, 30100 Ales, France \\ *pascale.montreer@mines-ales.fr; ${ }^{\circ}$ stephane.cariou@mines-ales.fr \\ ${ }^{2}$ IMT Mines Alès, LGI2P laboratory, 6 av. de Clavières, 30100 Ales, France \\ stefan.janaqi@mines-ales.fr \\ ${ }^{3}$ Olentica SAS, 17 rue Charles Peguy, F-30100 Ales, France \\ *mathilde.chaignaud@olentica.fr; jean-louis.fanlo@olentica.fr \\ ${ }^{4}$ Total S.A, 2, place Jean Millier, La Défense 6, 92078 Paris La Défense Cedex, France \\ *isabelle.betremieux@total.com; 'philippe.ricoux@total.com \\ ${ }^{5}$ Hutchinson S.A., Rue Gustave Nourry, 45120 Châlette-sur-Loing, France \\ frederic.picard@hutchinson.com \\ ${ }^{6}$ Total Feluy, Zone industrielle Feluy C, 7181, Belgique \\ *sabine.sirol@total.com; ${ }^{\circ}$ budagwa.assumani@total.com
}

\begin{abstract}
Odour control is an important industrial and economical issue as it is a criterion in purchase and use of a material. The minimal concentration of a pure compound allowing to perceive its odour, called Odour Detection Threshold (ODT), is a key parameter of the odour control. Each compound has its own ODT. Literature is the main source to obtain ODT. Nevertheless, there are a lot of compounds with no reported ODTs and when ODTs are available, they are marred by a high variability. Another expensive and time-consuming way to obtain ODT is the measurement. This paper proposes a validated cleaning methodology to reduce uncertainty of available ODTs. This methodology will be consolidated by our own experimental measurements. Next, we predict missing ODTs as a function of chemical and physical variables.

The proposed cleaning methodology leads to eliminate $39 \%$ of compounds with at least one ODT while conducting $84 \%$ of positive scenarios (on 37 compounds). The missing ODTs are predicted with an error of 0.83 for the train and 1.14 for the test (on a $\log 10$ scale). Given the uncertainty of data, the model is sufficient. This approach allows working with a lower uncertainty on available ODTs and predicts missing ODTs with a satisfactory model.
\end{abstract}

Keywords: Odour Detection Thresholds (ODT), Data mining, Reliability, Completeness, Uncertainty. 


\section{$1 \quad$ Introduction}

In the industrial environment, there is a growing need to identify compounds responsible for an unpleasant odour. This identification depends firstly on the Odour Detection Threshold (ODT) of each compound. We define ODT as the minimal concentration of a pure compound allowing to perceive its odour.

The principal source of ODTs is the literature $[1,2,3,4,5,6]$. But, in literature, there are a lot of compounds with no reported ODTs and when ODTs data are available, they present a high variability. This situation implies a high uncertainty of ODTs.

This variability can be illustrated with the butyl acetate example whose ODT values range from $0.030 \mathrm{mg} / \mathrm{m}^{3}$ to $480 \mathrm{mg} / \mathrm{m}^{3}$ for 14 publications [6].

The variability could potentially be explained by a set of parameters such as: difference of methods; existence and year of normalization; sample quality; environmental conditions, culture of authors; panel selection and their intrinsic diversity; panel correction, etc. Given all these potential sources of variability, there is a need to improve the reliability of these data. Unfortunately, the sparsity of these data makes most of the potential interesting statistical tools unusable. This sparsity comes from non-uniformity of the information from one author to another.

Several papers have already highlighted this issue [2,3] and have tried to compare publications [9] but usually, researchers make a subjective sorting or simply use the mean of values [3, 5]. But, in any event, even if dataset is used without cleaning, neither the mean or the geometric mean nor the density application is justified if the ODT values distribution is not identified.

As a consequence, our paper aims to find relevant methodology allowing improving the reliability of these data. This improvement gathers an approach aiming to decrease the variability of available ODTs by a cleaning methodology. Next, we complete not reported compounds by a predictive modelling of ODTs as a function of chemical / physical variables. Explicative variables generated by the methodology are precise opposed to bibliographic ODTs.

\section{$2 \quad$ Methodology}

\subsection{Software}

The ODT values from the literature are collected in an Excel sheet and the reliability improvement is realized on the $\mathrm{R}$ software.

\subsection{Database Construction}

To analyze ODT, a database is constructed (Fig. 1). This database gathers quite a few volatile and odorous compounds. These compounds constitute the rows of the database. 
For each compounds, a state of art of ODTs is done to identify publications containing ODT information [6]. These publications constitute the columns.

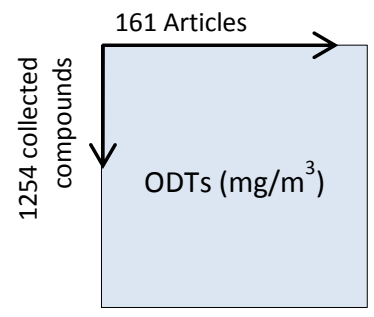

Fig. 1. ODT values $\left(\mathrm{mg} / \mathrm{m}^{3}\right)$ database format

This database will be the support of the uncertainty reduction and prediction. To date, it contains 161 publications (columns) and 1254 compounds (rows) including only 650 compounds with at least one ODT available.

\subsection{First Step of Cleaning: Provisional Publications' Isolation}

The first step consists in eliminating the least reliable studies. Ideally, it would have been relevant to consider only studies containing a reliable repeatability of measurements. In this way the Cochran test based on the standard deviation of each article would have been applied [11]. Unfortunately, this information is rarely available. Considering only studies with available repeatability is therefore too restrictive. Consequently, we use the criterion of number of ODT measurements realized per study. Indeed, we have noticed that the higher the number of ODT determinations in a publication, the better the technique of analysis was described, and hence the more reliable the results. It was decided to eliminate ten percent of the total values of the database and that corresponds to publications containing less than four ODT values (Fig. 2).

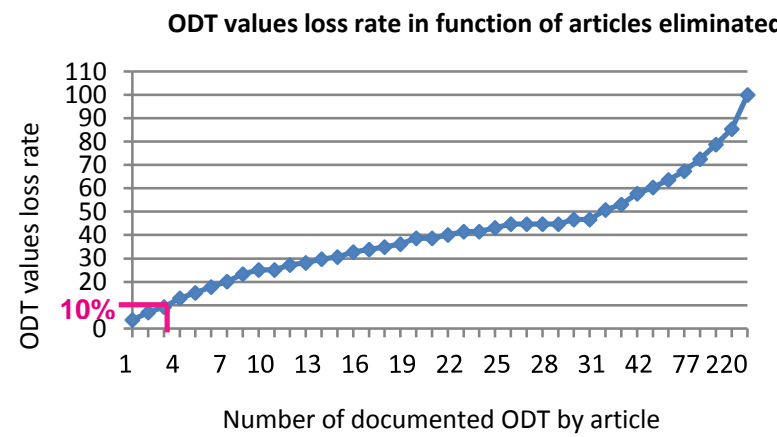

Fig. 2. ODT values loss rate in function of publications eliminated 


\subsection{Second Step of Cleaning Methodology: ODT Outlier Elimination}

\subsubsection{Outliers Definition in the Context}

To define an outlier in this context, the ODT values distribution has to be defined. On the basis of an expert observation on well-known molecules, the hypothesis is that for each given compound, ODTs follow a normal distribution.

Considering the low amount of ODT values per compound (Fig. 3), the most suitable solutions to observe this distribution, are the Kernel Density Estimation (KDE) and the QQplot representation of the eleven compounds with more than ten ODT values after the first step of the cleaning.

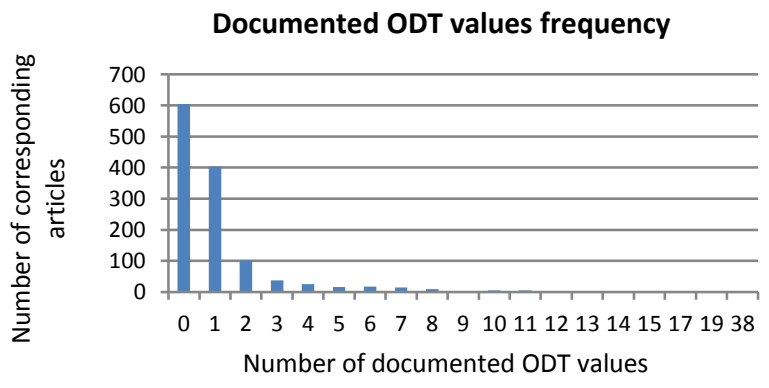

Fig. 3. Reported ODT frequency

The KDE is calculated with the "geom_density" function and the QQplot with "qqnorm" function. The correlation coefficient (CC) of QQplot of these compounds is calculated (Fig. 4).

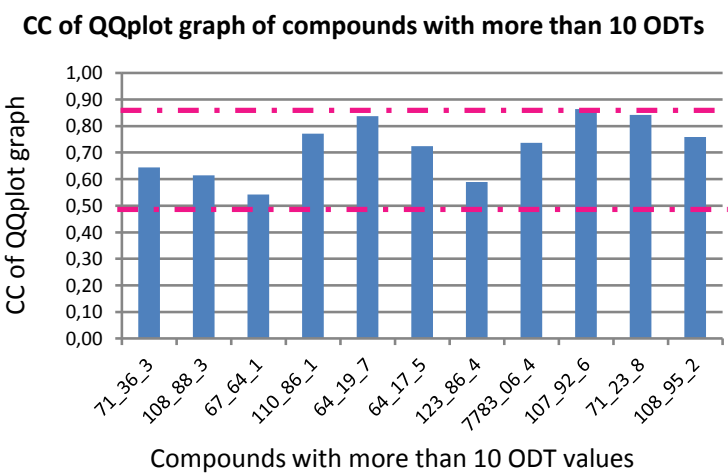

Fig. 4. Correlation coefficient of the QQplot of compounds with more than 10 ODT

On this Fig. 4 the higher the correlation coefficient is, the more likely the distribution is normal. These results encourage applying a normal test on values to detect outliers. 
The inter-laboratory reproducibility Grubbs test is applied [11]. The confidence level chosen is $95 \%$.

\subsubsection{Outliers Values Elimination According to Normal Distribution}

The Grubbs test is not applicable with less than three values [12]. In this way, only compounds measured more than twice can be compared with the rest of the database. Thereafter, ODTs of these compounds are considered as the "tested" ODTs. That implies that some compounds' relevance cannot be analyzed at this step.

Another rule of comparison has to be set up. This is an extension of the cleaning methodology to the elimination of publications outliers. This next rule was guided by the fact that, at the previous step, the eliminated ODT values often belong to the same publications.

\subsection{Third Step of Cleaning Methodology: Publication Outliers Elimination}

At this step, the relevance of each publication is measured by the number of exclusion of that article at the second step. An index is calculated for each author: the exclusion frequency ratio in equation (1).

$\mathbf{E F}_{j}=\frac{N_{\text {ODT excluded }}^{j}}{N_{\text {ODT tested }}^{j}} \times 100 \begin{aligned} & \boldsymbol{E F}_{\boldsymbol{j}} \\ & \boldsymbol{N}_{\text {ODT excluded }}^{j} \\ & \begin{array}{l}\text { Grubbs test } \\ \boldsymbol{N}_{\text {ODT tested }}^{j}\end{array}\end{aligned}:$ Number of ODT values of the jth publication excluded by

The ODT values of publications have not been compared in the same way. That's why a "tested rate" is calculated. For each publication, this "tested rate" is defined as the percentage of tested ODTs among all of the ODTs of this publication. Indeed, the higher the "tested rate" is; the better the $E F$ 's reliability is.

First, publications with "tested rate" under $1 / 3$ are eliminated. Then, after a Hierarchical Ascendant Classification (HAC) applied on the $E F$ of selected publications, the Ward's distance index [13] is used. This classification allows to statistically separate publications based on $E F$ values. The dendrogram and the SPRSQ graph are used to determine the number of groups. The group containing the lowest $E F$ is finally retained.

\subsection{Validation of the Cleaning}

ODT of 44 compounds were measured experimentally in our laboratory to validate the relevance of this cleaning. These ODT were measured using the norm EN 13725 [14]. For these 44 compounds, whose ODT values have been measured experimentally, 40 were reported in the literature. For these 40 compounds, three barycenters are defined: 
the one of raw ODT values of the literature (X1), the one of remaining ODT values after the cleaning methodology application (X2) and the one of our experimental ODT values (X3). And then two differences are calculated: X1-X3 (Z1) and X2-X3 (Z2). These two differences are compared. This comparison allows to observe if the cleaning methodology leads to approach the experimental result.

To state on the relevance of the cleaning the criterion to select the "ideal" case is the mean of the $\log 10$ of the confidence interval obtained thanks the repeatability level of our experimental measurements.

\subsection{Completeness of the Database by Predictive Modelling}

The completeness of the database is based on a Quantitative Structure-Property Relationship (QSPR) approach. This approach consists in predicting a variable $(\mathrm{Y})$ as a function of chemical and physical certain variables $(\mathrm{X})$. Here the $\mathrm{Y}$ variable is the mean value of $\log 10$ values of cleaned ODTs. Explicative variables $X$ are essentially calculated from the structure of compounds (66 variables). There are compositional and constitutional indexes (the number and nature of atoms, the molecular weight, the unsaturation), topological indexes which the majority are defined in Todeschini's publication [15] and electrotopological indexes as ZEP index [16].

The model approach is then divided in 2 principal steps. The first is a reduction of the number of significative input variables by a lasso technique. To predict $\mathrm{Y}$ as a function of X, a classical Support Vector Machine (SVM) model was constructed. Some "bad" observations was eliminated from this learning process.The partition of the dataset between the train and test sets is made in order that the train represents $75 \%$ of the dataset and reflects the variance of this one.

\section{$3 \quad$ Results and Discussion}

\subsection{Visualization of the Proportion of Missing Values of the Database}

Missing value proportion of the database (Fig. 1) is presented as a heatmap (Fig. 5). 


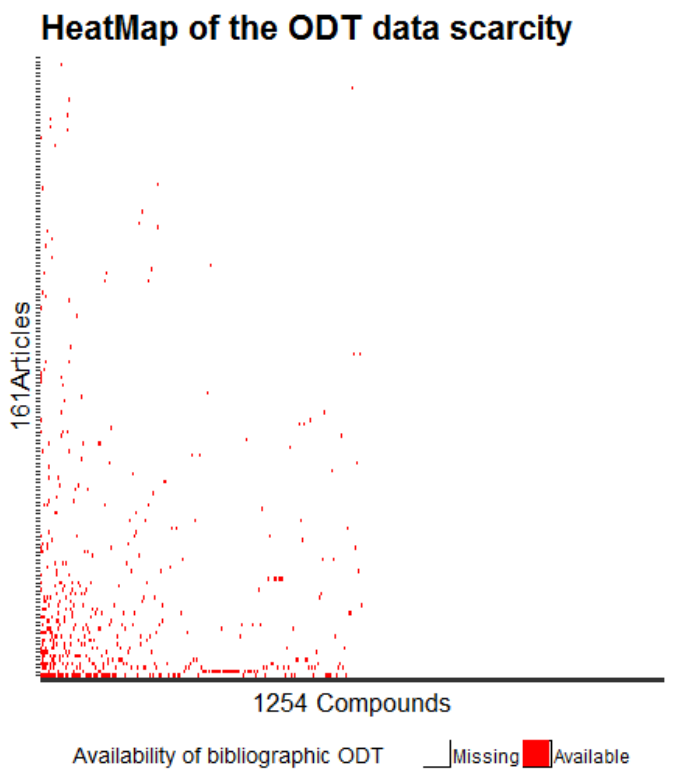

Fig. 5. Proportion of missing ODT values in the literature (a red zone corresponds to information presence; a white zone corresponds to a lack of information)

The total proportion of missing values is $99 \%$. This proportion enforces the inability to use statistical classification tools and implies the necessity to implement a more reliable methodology.

\subsection{Result of the First Step}

For the three "data cleaning" steps, information loss is presented from three perspectives (Table 1): the number of publications, the number of ODT and the number of compounds with at least one ODT. This choice is made because the most important information to monitor is the number of compounds with at least one ODT. Indeed, as it was presented, we want to predict the ODT behavior of all the compounds of the database (1254) with reported ones in literature. That's why, it's important to keep a satisfactory proportion of reported compounds.

Table 1. Information loss after the first step

\begin{tabular}{lccc}
\hline & \multicolumn{3}{c}{ Cleaning at the first step } \\
\hline & Before & After & Information loss \\
\hline Nos. of publications & 161 & 72 & $55 \%$ \\
\hline Nos. of ODT & 1501 & 1367 & $9 \%$ \\
\hline Nos. of compounds with at least 1 ODT & 650 & 631 & $3 \%$ \\
\hline
\end{tabular}


The first step leads to isolate $55 \%$ of the publications. Even if half of the publications have been eliminated, only $3 \%$ of compounds with at least one ODT have been eliminated. This proportion is acceptable. The lack of reliability on these sources justifies to reject them.

\subsection{Result of the Second Step}

The Grubbs test is applied on compounds which ODT have been measured at least 3 times. Results of the information loss of this step are presented in Table 2.

Table 2. Information loss after the second step by Grubbs test

\begin{tabular}{lccc}
\hline & \multicolumn{3}{c}{ Cleaning at the second step } \\
\hline & Before & After & Information loss \\
\hline Nos. of publications & 72 & 72 & $0 \%$ \\
\hline Nos. of ODT values & 1367 & 1235 & $10 \%$ \\
\hline Nos. of compounds with at least 1 ODT & 631 & 631 & $0 \%$ \\
\hline
\end{tabular}

There is only a slight loss of information on the ODT values (10\%) and the two other rates are still constant. Furthermore, among this proportion, there is an average of 1.65 values eliminated per compound (over 80 compounds). This low percentage supports the use of a normal distribution.

\subsection{Result of the Third Step}

A summary of ODT elimination at the second step on all the 72 publications, will allow applying the third step. The focus is made on the Exclusion Frequency (EF) defined by the equation (1) and the "tested rate" defined in the 2.5 section.

As it was mentioned in this section, publications with "tested rate" under $1 / 3$ are eliminated. A first information loss summary is done after the elimination of these publications with a "tested rate" lower than 33\% (Table 3).

Table 3. Information loss after the elimination of publicationss with tested rate lower than $33 \%$

\begin{tabular}{lccc}
\hline & \multicolumn{3}{c}{ Cleaning at the third step - Part 1 } \\
\hline & Before & After & Information loss rate \\
\hline Nos. of publications & 72 & 60 & $17 \%$ \\
\hline Nos. of ODT values & 1235 & 1064 & $14 \%$ \\
\hline Nos. of compounds with at least 1 ODT & 631 & 485 & $23 \%$ \\
\hline
\end{tabular}

The dendrogram of the HAC classification is applied on the EF of publications with a "tested rate" higher than $33 \%$ (Fig. 6). 


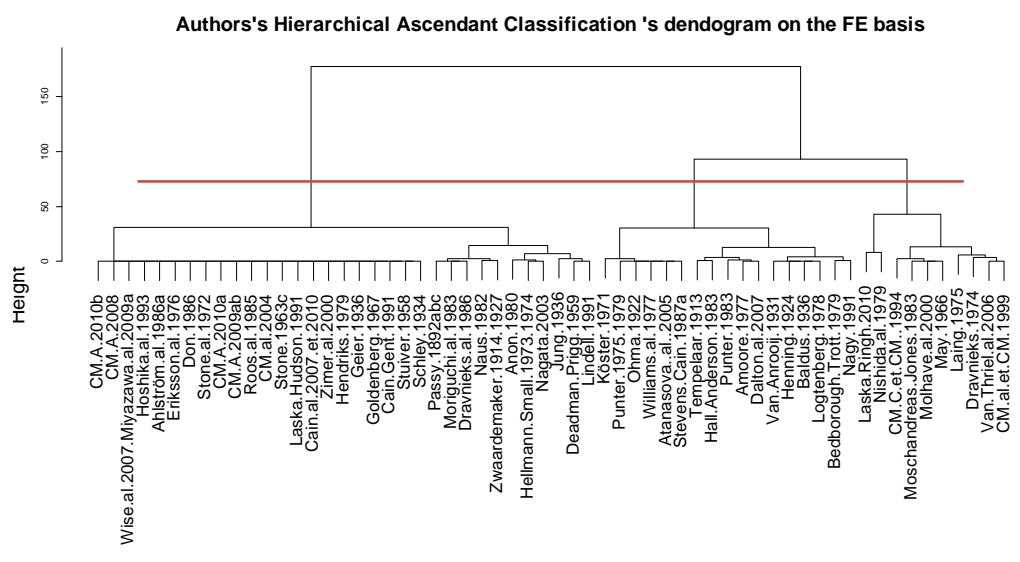

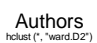

Fig. 6. HAC on EF values dendrogram (red line: the optimal classification)

Thanks to the dendrogram and the SPRSQ graph, 3 groups were realized (Fig. 6). The information loss summary is done after the second part of the third step (Table 4).

Table 4. Information loss after the third step

\begin{tabular}{lccc}
\hline & \multicolumn{3}{c}{ Cleaning at the third step - Part 2 } \\
\hline & Before & After & Information loss \\
\hline Nos. of publications & 60 & 33 & $45 \%$ \\
\hline Nos. of ODT values & 1064 & 701 & $34 \%$ \\
\hline Nos. of compounds with at least 1 ODT & 485 & 393 & $19 \%$ \\
\hline
\end{tabular}

This third step is rather drastic because it eliminates $37.7 \%$ of compounds with at least one reported ODT value in the literature.

Nevertheless, 393 reported compounds still remain, spread over 33 publications. The size of the database decreases with the increase of its reliability. Of course, we consider that it is better to work with less quantitative but more informative data.

\subsection{Summary of the Cleaning Methodology}

After the three cleaning methodology steps, the information loss statement is presented in Table 5.

Table 5. Information loss statement after three steps of the cleaning methodology

\begin{tabular}{lccc}
\hline & \multicolumn{3}{c}{ Total cleaning } \\
\hline & Before & After & Total information loss \\
\hline Nos. of publications & 161 & 33 & $79 \%$ \\
\hline Nos. of ODT values & 1501 & 701 & $53 \%$ \\
\hline Nos. of compounds with at least 1 ODT & 650 & 393 & $\mathbf{3 9 \%}$ \\
\hline
\end{tabular}


It can be noticed that a high percentage of publications is eliminated (79\%) but it represents almost the double of compounds with at least one ODT value in the literature (39\%). The amount of total eliminated data is $53 \%$. These rejected data have been considered irrelevant by our specific approach.

\subsection{Validation of the Cleaning Methodology with Measured ODT}

After the cleaning, the ODT value of 3 compounds out of 40 was totally eliminated and we make the comparison on the 37 remaining compounds. The mean of the repeatability of our measurement calculated in a $\log 10$ scale is 0.4 . The ODTs obtained are then compared to this value as it was explained in the 2.6 section (Fig. 7).

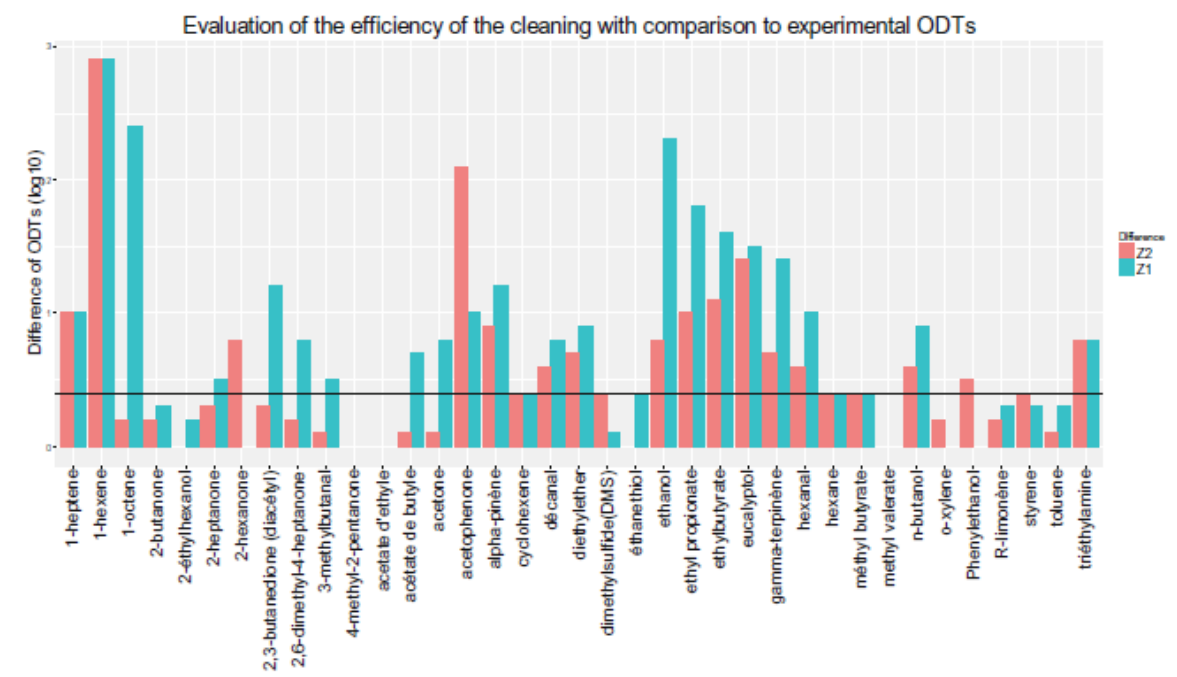

Fig. 7. Comparison of the cleaned values and the raw values of literature with our experimental values (red: cleaned ODT difference; blue: raw ODT difference)

Many scenarios are recorded (Table 6) thanks to the graph of comparisons (Fig. 7).

Table 6. Summary of the evolution by the cleaning methodology

\begin{tabular}{|c|c|c|c|}
\hline Scenario 1 & \multirow{3}{*}{ Improvement of the value ... } & ... even if it was already correct & 5 cases \\
\hline Scenario 2 & & ... which leads to a correct value & 6 cases \\
\hline Scenario 3 & & $\ldots$ but the value still not correct & 10 cases \\
\hline Scenario 4 & \multirow[t]{2}{*}{ No improvement of the value } & ... but it was already correct & 7 cases \\
\hline Scenario 5 & & $\ldots$ and still not correct & 3 cases \\
\hline Scenario 6 & \multirow{3}{*}{ Degradation of the value ... } & ... but it still correct & 3 cases \\
\hline Scenario 7 & & ... but it still not correct & 1 cases \\
\hline Scenario 8 & & ... becomes not correct & 2 cases \\
\hline
\end{tabular}


Initially, 16 compounds were in the range of the measured ODT. After the cleaning, there are 21 compounds (out of 37 compared compounds). This is a first positive added value of the cleaning methodology.

Furthermore, it can be considered as positive situation after the cleaning the scenarios 1, 2, 3, 4 and 6 (Table 6). These cases represent $84 \%$ of positive cases (31 compounds out of 37). The worst scenario is the number 8 . Despite of 6 compounds, this cleaning seems appropriate to decrease the uncertainty of available bibliographic ODT data.

\subsection{ODTs Prediction}

Applying lasso technique leads to eliminate 22 variables (out of 66) and SVM leads to a model with an error, in a $\log 10$ scale, of 0.83 on the train and 1.14 on the test with compounds eliminated (Fig. 8). As a matter of fact, among all the predictions, only less than $2.5 \%$ of observations was abnormally predicted in comparison with the others.

ODTs predicted in fucntion of ODTs observed

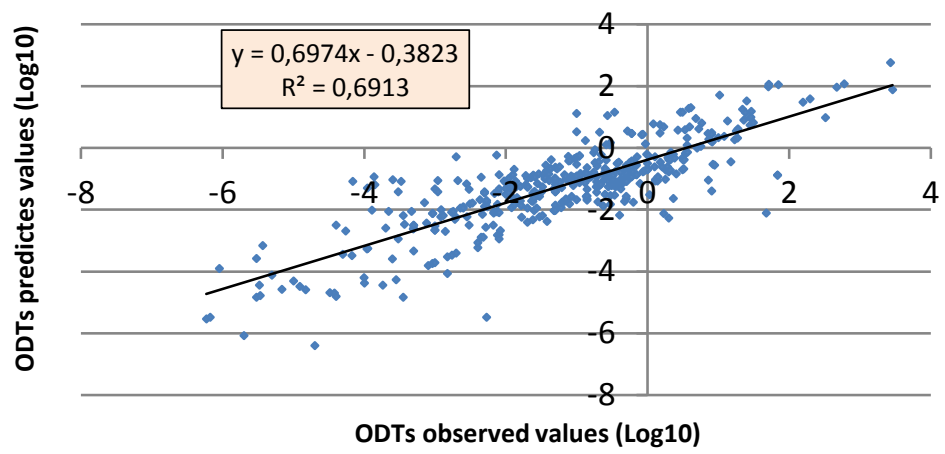

Fig. 8. ODTs predicted in function of ODTs observed

Given the uncertainty of ODT of most of compounds, the model is satisfactory as it predicts the ODT with a $\log 10$ error about 1 . To our knowledge this is the first global model to predict ODT as a function of molecular characteristics on this quantity of compounds. This is an encouraging result which has to however be improved thereafter.

\section{Conclusion}

In conclusion, the proposed cleaning methodology eliminates $39 \%$ of compounds with at least one ODT while conducting to $84 \%$ of positive scenarios on ODT values (validation on 37 compounds). The missing ODTs are predicted with an error of 0.83 for the train and 1.14 for the test (on a $\log 10$ scale). Considering the data, it's a satisfactory model. This approach allows working with a low uncertainty on available ODTs and predicts missing ODTs with a satisfactory model. This is an encouraging methodology. 
Thereafter, it would be interesting to strengthen the cleaning methodology with more measurements in our laboratory or a probabilistic validation.

The global predicting model of ODT seems satisfactory to odour experts. It will be improved further by complementary local approach and / or expert knowledge

\section{$5 \quad$ References}

1. LEONARDOS, G., KENDALL, D., BARNARD, N.: Odor Threshold Determinations of 53 Odorant Chemicals. Journal of the Air Pollution Control Association. 19, 91-95 (1969). doi:10.1080/00022470.1969.10466465

2. RUTH, J.H.: Odor Thresholds and Irritation Levels of Several Chemical Substances: A Review, (1986)

3. DEVOS, M., PATTE, F., LAFFORT, P., VAN GEMERT, L.J.: Standardized human olfactory thresholds. Oirl Press (1990)

4. ASTM: Odor Thresholds for Chemicals with Established Occupational Health Standards. (1997)

5. US EPA: Reference guide to odor thresholds for hazardous air pollutants listed in the clean air act amendements of 1990. (1992)

6. VAN GEMERT, L.J.: Odour Thresholds., Zeist, The Netherlands (2011)

7. FALCY, M., MALARD, S.: Comparaison des seuils olfactifs de substances chimiques avec des indicateurs de sécurité utilisés en milieu professionnel. Hygiène et Sécurité au travail- Cahier de notes documentaire. 7-21 (2005)

8. ZARZO, M.: Effect of Functional Group and Carbon Chain Length on the Odor Detection Threshold of Aliphatic Compounds. Sensors. 12, 4105-4112 (2012). doi:10.3390/s120404105

9. PATTE, F., ETCHETO, M., LAFFORT, P.: Selected and standardized values of suprathreshold odor intensities for 110 substances. Chemical Senses and Flavor. 283-305 (1975)

10. TOROPOV, A.A., TOROPOVA, A.P., CAPPELLINI, L., BENFENATI, E., DAVOLI, E.: Odor threshold prediction by means of the Monte Carlo method. Ecotoxicology and Environmental Safety. 133, 390-394 (2016). doi:10.1016/j.ecoenv.2016.07.039

11. ISO17025: DémarcheISO17025, marcheiso17025.com/fiches_techniques/test_grubbs_cochran.html

12. ASTM International: ASTM E178-00 Standard practice for dealing with outlying observation. (1994)

13. WARD, J.H.J.: Hierarchical grouping to optimize an objective function. Journal of the American Statistical Association. 58, 236-244 (1963)

14. AFNOR: Norme NF EN 13725. (2003)

15. TODESCHINI, R., CONSONNI, V., MANNHOLD, R., KUBINYI, H., FOLKERS, G.: Molecular Descriptors for Chemoinformatics. (2009)

16. BERINDE, Z., BERINDE, M.: On a matrix representation of molecular. CARPATHIAN J. MATH. 20, 205-209 (2004) 